\title{
FIR/Sub-mm Line Emission from the First Objects: Testing the Stellar Feedback
}

\author{
Benedetta Ciardi ${ }^{1}$ and Andrea Ferrara ${ }^{2}$ \\ 1 Universitá di Firenze, Largo Enrico Fermi 5, 50125 Firenze, Italy \\ 2 Osservatorio Astrofisico di Arcetri, Largo Enrico Fermi 5, 50125 Firenze, Italy
}

May 2000

\begin{abstract}
We calculate the expected FIR/sub-mm molecular hydrogen line emission from the first galactic objects that formed in the universe. Due to their low masses, the stellar feedback from massive stars is able to blow away their gas content and collect it into a cooling shell where $\mathrm{H}_{2}$ rapidly forms and IR rotational lines carry away a large fraction of the explosion energy. The expected fluxes from these sources are in the range 10$100 \mu \mathrm{Jy}$. The highest number counts are expected in the $60-80 \mu \mathrm{m}$ band, where about 1000 sources $/ \mathrm{deg}^{2}$ are predicted at the limiting flux of $7 \times 10^{2} \mu \mathrm{Jy}$. Among the planned FIR/sub-mm facilities, we find that the best detection perspectives are offered by the Multiband Imaging Photometer for SIRTF, which should be able to reveal about 3500 first objects in one hour observation time. Therefore, FIR/sub-mm instruments appear to represent perfect tools to trace star formation and stellar feedback in the very high $(z \gtrsim 10)$ redshift universe.
\end{abstract}

Key words: galaxies: formation - intergalactic medium - cosmology: theory - ISM: molecules

\section{INTRODUCTION}

Detecting the first objects that formed in the universe will be the primary goal of several future space- and ground-based telescopes. The importance of these experiments consists in the fact that they could be able to test current cosmological scenarios, study the properties of these (supposedly very small) galaxies, and their effects on the surrounding environment (as for example reionization, heating and metal enrichment of the IGM). Due to the predicted low luminosity of these objects, this task will be at the capability edge of even the most advanced and powerful instruments. Apart from the indirect probes of their effects, as for example the secondary anisotropies in the CMB left by reionization (Knox, Scoccimarro \& Dodelson 1998; Gruzinov \& Hu 1998; Bruscoli et al. 2000; Benson et al. 2000), some search strategies have already been suggested in the literature. Marri \& Ferrara (1998) and Marri, Ferrara \& Pozzetti (2000) have suggested that Type II supernovae occurring in the first objects might outshine their parent galaxy by more than a hundred times and become visible by instruments like the Next Generation Space Telescope (NGST). Oh (1999) proposes to directly imaging the ionized halos around primordial objects either via their free-free emission (possibly detectable with the Square Kilometer Array) or Balmer line emission again with NGST.
One point that is particularly important when dealing with these low mass systems is that they are strongly affected by feedback mechanisms both of radiative and stellar type; these have been extensively investigated by Ciardi et al. (2000). Molecular hydrogen, being the only available coolant in a plasma of nearly primordial composition, is a key species in the feedback network as it regulates the collapse and star formation in these objects. Ferrara (1998, F98) has pointed out that $\mathrm{H}_{2}$ is efficiently formed in cooling gas behind shocks produced during the blowaway (i.e. the complete ejection of the galactic gas) process thought to occur in the first objects, with typical fractions of $f_{H_{2}} \approx 6 \times 10^{-3}$. The conditions in these cooling blastwaves are such that a noticeable amount of the explosion energy is carried away by infrared (redshifted into the FIR/sub-mm band) $\mathrm{H}_{2}$ molecular lines, which therefore might provide us with a superb tool to detect and trace these very distant primordial galactic blocks.

The use of molecular lines as diagnostics of moderate redshift $(z \lesssim 3)$ galaxies has already been proposed by some authors (Frayer \& Brown 1997; Blain et al. 2000); at higher redshifts pioneering calculations were carried on by Shchekinov \& Éntel' (1985); more recently Silk \& Spaans (1997) concentrated on the CO and dust emission from HII regions inside larger galaxies. All these studies have emphasized the power of the molecular line emission as a probe 
of distant sources. In this paper we calculate the expected FIR/sub-mm fluxes and number counts for these sources in various bands and assess if forthcoming observing facilities will be able to eventually unveil the beginning of the cosmic star formation process and study the feedback processes in the young universe.

\section{EMISSION MODEL}

Due to its symmetry, the $\mathrm{H}_{2}$ molecule has no electric dipole moment in the ground state. Therefore, the first detectable $\mathrm{H}_{2}$ emission lines are produced by quadrupole radiation and they are purely rotational. The lines relevant to the present work are listed in Table 1.

The temperatures required to excite these lines fall in the range 500-4500 K. The temperature range spanned during the cooling of the post-shock IGM gas produced during the blowaway of low-mass primordial galaxies is (F98)

$300 \mathrm{~K} \lesssim T \lesssim 10^{-3}(1+z)^{21 / 5} M_{6}^{2 / 5} \mathrm{~K}$ for $\quad(1+z) \geqslant 6.7,(1)$

where $M=10^{6} M_{6} M_{\odot}$ is the total mass of the galaxy. ${ }^{\star}$ The redshift constraints in the previous equation comes from the requirement that the blastwave becomes radiative, i.e. the cooling time is shorter than the Hubble time at the explosion epoch. It is then conceivable to expect that the above molecular lines are excited during this process, producing potentially detectable radiation. The predicted flux on the ground in a given line is derived as follows.

Let $\nu_{i k}$ be the restframe frequency of photons emitted by the molecule during the transition between the energy levels $k$ and $i$ then the line emissivity is (Spitzer 1978):

$j_{\nu_{i k}}=\frac{h}{4 \pi} \nu_{i k} n_{k} A_{k i} \Phi\left(\nu_{i k}\right)$

where $n_{k}$ is the number density of molecules in the $k$ level, $A_{k i}$ is the Einstein coefficient for spontaneous emission and $\Phi\left(\nu_{i k}\right)$ is the line profile function calculated at the line center. As the typical $\mathrm{H}_{2}$ densities found are much lower than the critical one $\left(\approx 10^{4} \mathrm{~cm}^{-3}\right)$ we neglect collisional de-excitations. If the line profile is dominated by Doppler broadening, and the velocity distribution is Maxwellian, this can be written as

$\Phi\left(\nu-\nu_{i k}\right)=\frac{1}{\sqrt{\pi} \Delta \nu_{D}} \mathrm{e}^{-\left(\nu-\nu_{i k}\right)^{2} / \Delta \nu_{D}^{2}}$,

with $\Delta \nu_{D}=\left(\nu_{i k} / c\right) \sqrt{2 k T / m}$, where $m$ is the molecular mass.

As the conditions for thermodynamic equilibrium are not satisfied, the population of the various levels must be obtained by solving the detailed balance equations (Spitzer 1978). Using this approach, the number density of molecules in the vibrational level $v, n_{J}$, of even and odd rotational levels decouple and they are obtained by iteration using the following formula:

$n_{J+2}(v)=n_{J}(v) g_{J} \frac{\gamma_{J} \mathrm{e}^{-\Delta E / k T}}{1+\gamma_{J}}$,

* We adopt a $\Lambda$ CDM (cluster normalized) cosmology with $\Omega_{M}=$ $0.35, \Omega_{\Lambda}=0.65, \Omega_{b}=0.04, h=0.65$.
Table 1: Relevant $\mathrm{H}_{2}$ Transition Lines

\begin{tabular}{lccc}
\hline \hline Transition & $\Lambda^{(1)}[\mu \mathrm{m}]$ & $\mathrm{T}^{(2)}[\mathrm{K}]$ & $\mathrm{A}^{(3)}\left[\mathrm{s}^{-1}\right]$ \\
\hline \hline $0-0 \mathrm{~S}(0)$ & 28.0 & 512 & $2.94 \times 10^{-11}$ \\
$0-0 \mathrm{~S}(1)$ & 17.0 & 1015 & $4.76 \times 10^{-10}$ \\
$0-0 \mathrm{~S}(2)$ & 12.3 & 1681 & $2.76 \times 10^{-9}$ \\
$0-0 \mathrm{~S}(3)$ & 9.7 & 2503 & $9.84 \times 10^{-9}$ \\
$0-0 \mathrm{~S}(5)$ & 6.9 & 4586 & $5.88 \times 10^{-8}$ \\
\hline
\end{tabular}

(1) Emission wavelength

(2) Excitation temperature (Combes \& Pfenninger 1998; Timmermann et al. 1996)

${ }^{(3)}$ De-excitation Einsten coefficient (Turner, Kirby-Docken \& Dalgarno 1977)

where $g_{J}$ is the statistical weight of level $J, \gamma_{J}$ is the ratio between the collisional excitation rate and the Einstein $A$ coefficient, $\Delta E$ is the energy difference between the two levels. Obviously, in the limit of large $\gamma_{J}$ eq. 4 approaches the Boltzmann distribution.

Given the post-shock temperatures found, it is only necessary to consider the two vibrational levels $v=0,1$. The relative number of molecules in these two levels depends on the total molecular hydrogen density, $n_{H_{2}}$. For a given redshift, the value of $n_{H_{2}}$ (F98) is:

$n_{H_{2}}(z)=p f_{H_{2}}\left(M_{6}, z\right) n_{s} \simeq p f_{H_{2}}\left(M_{6}, z\right) \delta n_{H}(z)$,

where $f_{H_{2}}$ is the molecular fraction in the shell, and $n_{H}(z)$ is the IGM hydrogen density. We allow for a density enhancement in the shell with respect to $n_{H}$ equal to $\delta$; this is produced both by the shock compression and the possible occurrence of the explosion inside an overdense region of the universe (e.g. a cosmological filament). Because of the first effect, and as the expanding blastwave becomes rapidly radiative, $\delta$ is given by the square of the shock Mach number, $\mathcal{M}$, with respect to the ambient IGM. Following F98, it is easy to show that the density enhancement is given by:

$\delta=12(1+z)^{13 / 5} M_{6}^{2 / 5}$ for $(1+z) \geqslant 6.7$.

For simplicity, we have neglected the additional density increase produced by an explosion in a overdense region; thus, the results presented above should be interpreted as a conservative lower limit to the detectability of the sources under investigation. Finally, we assume a branching ratio $p=0.75$ (0.25) for ortho (para) transitions which is valid under LTE conditions; however, non-equilibrium conditions might lead to slightly lower values of the ortho-to-para ratio (Chrysostomou et al. 1993; Rodríguez-Fernández et al. 2000).

With the emissivity in eq. 2, the observed flux is:

$F\left(\nu_{o}\right)=\frac{L_{\nu_{i k}}(1+z)}{4 \pi d_{L}^{2}}$

where $\nu_{o}=\nu_{i k} /(1+z)$ is the observed frequency, $L_{\nu_{i k}}$ the specific luminosity and $d_{L}$ is the cosmological luminosity distance. For a flat universe $\left(\Omega_{0}=\Omega_{M}+\Omega_{\Lambda}=1\right), d_{L}$ is given by

$d_{L}=(1+z) \int_{0}^{z}\left(1+z^{\prime}\right)\left|\frac{d t}{d z^{\prime}}\right| d z^{\prime}$,

$\left|\frac{d t}{d z}\right|^{-1}=H_{0}(1+z) \sqrt{\left(1+\Omega_{M} z\right)(1+z)^{2}-\Omega_{\Lambda} z(2+z)}$, 
where $H_{0}=100 h \mathrm{~km} \mathrm{~s}^{-1} \mathrm{Mpc}^{-1}$ is the current Hubble constant. The corresponding specific luminosity is:

$L_{\nu_{i k}}=4 \pi V j_{\nu_{i k}}$,

where $V=4 / 3 \pi R_{s}^{2} d R_{s}$ is the physical volume occupied by the $\mathrm{H}_{2}$ forming shell. The values of $R_{s}$ and $d R_{s}$ are obtained from the formulae in F98; the only difference consists in the assumption of a single burst of star formation, as opposed to the quiescent star formation prescription of F98. By assuming a star formation efficiency of $10 \%$, a SN every 100 $M_{\odot}$, it is easy to show that the total energy of the explosion is equal to $E=1.3 \times 10^{53} M_{6} \mathrm{erg}$. Then, from F98 it follows

$R_{s} \simeq 1.4 M_{6}^{1 / 5}(1+z)^{-11 / 5} \mathrm{Mpc}$,

$d R_{s}=\frac{N_{H_{2}}}{n_{H_{2}}} \simeq \frac{f_{H_{2}} M_{s}}{m_{H} R_{s}^{2} n_{H_{2}}}=\frac{4 \pi}{3 \delta p} R_{s}$,

where we have assumed that all the swept IGM mass is in the cool shell together with the galaxy ISM.

\section{NUMBER COUNTS}

The number of objects whose observed flux is larger than the threshold value $F_{\min }$ is:

$$
\begin{aligned}
N\left(>F_{\min }\right) & =\int_{z_{\min }}^{z_{\max }} d z \frac{d V}{d z} \int_{L_{\min }}^{L_{\max }} d L \frac{d n}{d M} \frac{d M}{d L} \\
& =\int_{z_{\min }}^{z_{\max }} d z \frac{d V}{d z} \int_{M_{\min }}^{M_{\max }} d M \frac{d n}{d M}
\end{aligned}
$$

where $d n / d M$ is the comoving number density of halos with masses between $M$ and $M+d M$ (Press \& Schechter 1974) and $d V / d z$ is the comoving volume element per unit redshift:

$\frac{d V}{d z}=\frac{4 \pi c d_{L}^{2}}{(1+z)}\left|\frac{d t}{d z}\right|$,

where $d_{L}$ and $d t / d z$ are given in eqs. 8 and 9 , respectively. The limits $z_{\min }$ and $z_{\max }$ depend on the observational wavelength band; $L_{\max }$ is the luminosity corresponding to the maximum mass value, $M_{\max }$, experiencing blowaway at redshift $z$ (Ciardi et al. 2000); $M_{\min }=\min \left[M_{H}, M\left(L_{m i n}\right)\right]$. Here $M_{H}$ is the minimum halo mass in which stars can form at a given redshift, which is related to the minimum virial temperature of $\approx 10^{4} \mathrm{~K}$ below which atomic hydrogen cooling of the gas is suppressed and fragmentation into stars is inhibited (Haiman, Rees \& Loeb 1997; Ciardi et al. 2000); $M\left(L_{\text {min }}\right)$ is the mass of the halo producing the luminosity $L_{\text {min }}$ corresponding to the observed flux $F_{\text {min }}$. Finally, $L_{\text {min }}$ is estimated as the mean of the integrated energy emission over the lifetime of the shock.

\section{RESULTS}

The model developed above allows us to determine the observational properties of the FIR/sub-mm emission from the first objects. In particular we present results concerning the expected flux in the various lines considered (see Table 1) and the corresponding number counts. These are then compared with the sensitivities of various planned instruments.

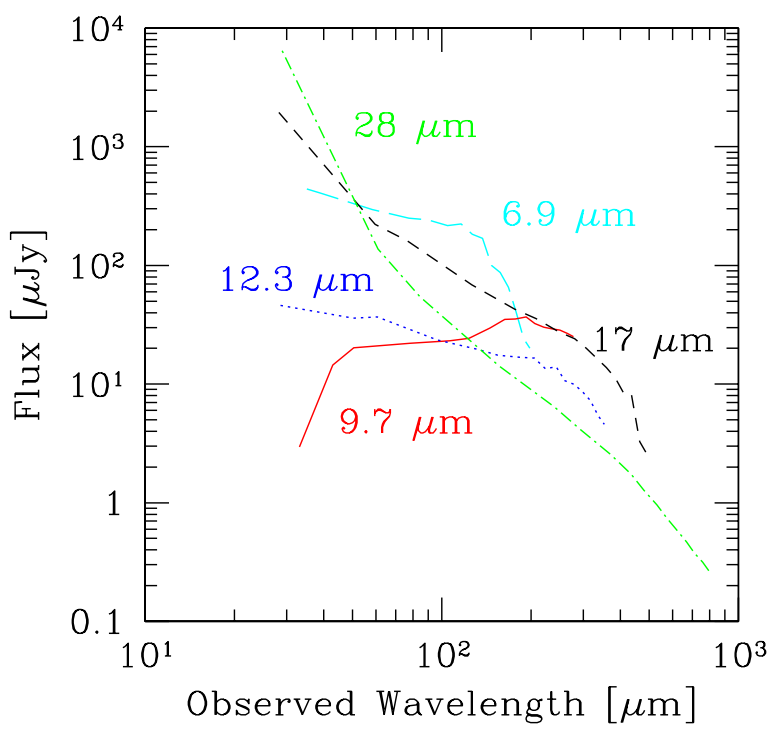

Figure 1. Expected flux as a function of the observed wavelength for the five $\mathrm{H}_{2}$ molecular lines considered: $6.9 \mu \mathrm{m}$ (long-dashed line), $9.7 \mu \mathrm{m}$ (solid), $12.3 \mu \mathrm{m}$ (dotted), $17 \mu \mathrm{m}$ (short-dashed) and $28 \mu \mathrm{m}$ (dot-dashed). The flux is derived for the average halo mass evaluated at the emission redshift (see text for details).

\subsection{Expected FIR/Sub-mm Fluxes}

Fig. 1 shows the expected flux as a function of the observed wavelength, $\lambda_{o b s}$, in the five different lines $6.9 \mu \mathrm{m}, 9.7 \mu \mathrm{m}$, $12.3 \mu \mathrm{m}, 17 \mu \mathrm{m}$, and $28 \mu \mathrm{m}$. As an example, we plot the fluxes calculated for the mass of the halos that suffer blowaway averaged over the halo mass distribution, as given by the Press-Schechter formalism, at the explosion redshift. In addition, the curves refer to emission occurring when the shocked gas temperature has decreased down to the corresponding line excitation temperature. This obviously implies that the emission redshift is lower than the explosion redshift.

From Fig. 1 we see that the typical observed fluxes are in the range $10-100 \mu \mathrm{Jy}$, depending on the wavelength band (and therefore emission redshift). For the three high excitation lines the flux is relatively constant with wavelength (i.e. emission redshift), whereas the flux of the two low excitation lines is rapidly decreasing towards higher $\lambda_{o b s}$. ¿From a general point of view this can be understood as follows. As the IGM density increases with redshift, this causes a corresponding increase in the emissivity; this effect is overwhelmed by a larger value of $d_{L}$ and a smaller shell emission volume. In addition, the postshock gas tends to be warmer at high $z$, hence inducing preferentially the excitation of the shorter wavelength lines. These two effects tend to compensate for the high excitation lines yielding a basically flat spectrum; for the low excitation lines instead the two effects sum together and produce a rapidly decreasing intensity.

\subsection{Number Counts and Detectability}

By using the expressions given in eq. 13 we can now calculate the expected number of objects per unit sky area as a 


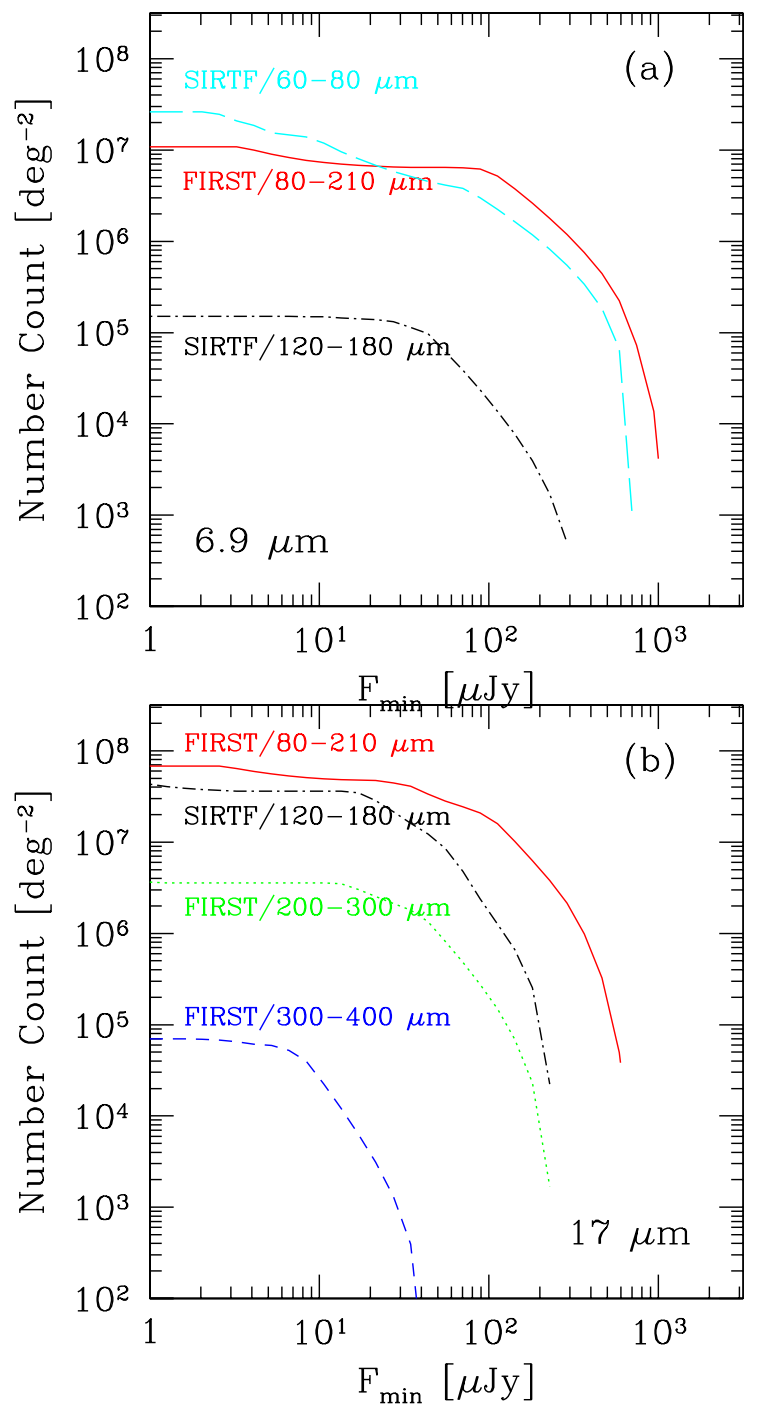

Figure 2. (a) Number counts from the $\mathrm{H}_{2} 6.9 \mu \mathrm{m}$ emission wavelength as a function of the minimum observed flux, $F_{\text {min }}$. The number counts are calculated for different observed wavelength bands: $60-80 \mu \mathrm{m}$ (long-dashed line), $80-210 \mu \mathrm{m}$ (solid) and 120$180 \mu \mathrm{m}$ (dot-dashed). (b) As Fig. (a) for the $\mathrm{H}_{2} 17 \mu \mathrm{m}$ emission wavelength. The number counts are calculated for: $80-210 \mu \mathrm{m}$ (solid line), 120-180 $\mu \mathrm{m}$ (dot-dashed), 200-300 $\mu \mathrm{m}$ (dotted), 300$400 \mu \mathrm{m}$ (short-dashed) band.

function of the limiting flux, $F_{\text {min }}$, of a given experiment. These are shown in Fig. 2 for the $6.9 \mu \mathrm{m}$ and $17 \mu \mathrm{m} \mathrm{H} \mathrm{H}_{2}$ lines. The reason for this choice is evident from inspection of Fig. 1, where it is seen that the band-integrated received energy in these lines is the highest among those considered. The number counts have been estimated in five different FIR/sub-mm bands corresponding to the sensitivity ranges of instruments on board of the planned space missions Space InfraRed Telescope Facility $\dagger^{\dagger}$ (SIRTF) and Far InfraRed and

$\dagger_{\text {http://sirtf.caltech.edu }}$
Submillimeter Telescope $e^{\ddagger}$ (FIRST). These bands (also indicated in Fig. 2) cover the wavelength range $60-400 \mu \mathrm{m}$. For the SIRTF $60-80 \mu \mathrm{m}$ band, the number counts for the $6.9 \mu \mathrm{m}$ line extend up to $7 \times 10^{2} \mu \mathrm{Jy}$ : at this flux level about 1000 objects $/ \mathrm{deg}^{2}$ are predicted. This value increases rapidly as $F_{\min }$ decreases and reaches a plateau $\left(\approx 3 \times 10^{7} / \mathrm{deg}^{2}\right)$ at about $1 \mu \mathrm{Jy}$. A second promising band is the FIRST $80-210$ $\mu \mathrm{m}$ one: both for the $6.9 \mu \mathrm{m}$ and the $17 \mu \mathrm{m}$ emission, the number counts extend up to $10^{3} \mu \mathrm{Jy}$ and $6 \times 10^{2} \mu \mathrm{Jy}$, respectively. Other bands show similar shapes, but they are shifted toward lower fluxes/counts.

We can now compare the above results with the foreseen capabilities of SIRFT and FIRST; all sensitivities are given for a $3 \sigma$ detection in one hour. The Multiband Imaging Photometer for SIRTF (MIPS) is expected to reach a sensitivity of $3 \times 10^{2} \mu \mathrm{Jy}$ in the band $60-80 \mu \mathrm{m}$ and of $5 \times 10^{3} \mu \mathrm{Jy}$ in the band $120-180 \mu \mathrm{m}$. At the above sensitivity level, and with its field of view of $5^{\prime} \times 5^{\prime}$, MIPS should be able to detect about 3500 objects in one hour integration at the $3 \sigma$ level. This is marginally above the confusion limit of the instrument and therefore the objects should be seen as discrete sources. The lower MIPS sensitivity in the longer wavelength band 120-180 $\mu \mathrm{m}$ essentially prevents detections. The PACS photometer on board of FIRST (sensitivity range $80-210 \mu \mathrm{m}$ ) will reach a limiting flux of $1.8 \times 10^{3} \mu \mathrm{Jy}$, and therefore can only detect emission from the $6.9 \mu \mathrm{m}$ line very marginally. Finally, SPIRE (Spectral and Photometric Imaging Receiver on board of FIRST) comprises a three band imaging photometer covering the $200-600 \mu \mathrm{m}$ range, where it is expected to reach a sensitivity of $\approx 4-5 \times 10^{3} \mu \mathrm{Jy}$, optimized in the short wavelength region of the band.

In conclusion, SIRTF/MIPS appear to constitute the best tool to reveal the emission from the first objects; other above mentioned instruments can also be used but they require much longer exposure times.

\section{CONCLUSIONS}

We have investigated the possibility to detect the first objects in the universe through the FIR/sub-mm line emission associated with the stellar feedback (i.e. blowaway of the gas) induced by their supernovae. A noticeable fraction of the explosion energy is eventually converted and carried away by molecular hydrogen rotational lines, a key species in the early universe. By using a detailed treatment of the non-equilibrium formation and emission of $\mathrm{H}_{2}$ molecules behind these cosmological blastwaves we have calculated the observed fluxes and number counts of primordial objects and compared them with the expected sensitivities of future instruments as SIRTF and FIRST. At the limiting flux of SIRTF/MIPS, we do predict that about 3500 primordial objects can be detected in the $60-80 \mu \mathrm{m}$ band in one hour of observation time.

This detection would allow to directly image the first star formation in the universe and our results show that FIR/sub-mm observations, when compared with estimates of previous studies in other bands and/or exploiting different strategies, are probably the best tool for this aim. In

$\ddagger$ http://astro.estec.esa.nl/First 
addition, the proposed excitation mechanism will also allow to test and calibrate the stellar feedback process in the dark ages and finally assess the degree at which the first galaxies (and the IGM) have been influenced by supernova energy deposition. This obviously holds the key for the understanding of the subsequent evolution and formation of larger galactic blocks in most cosmological models. FIR/Sub-mm line emission allows the very high redshift universe $(z \gtrsim 10)$ to be much more easily explored than in the near IR bands, where the intergalactic absorption might be found to strongly affect the detectability of objects located close or beyond the reionization epoch. It is worth noting that our predictions constitute lower limits to the number of observable objects as we have not considered possible density enhancements descending from explosions occurring inside overdense regions, as the filaments of the cosmic web.

Molecular hydrogen lines might also be excited during the collapse of the so-called PopIII objects, which rely on this molecule to collapse and form stars. However, there are several reasons to suspect that the contribution of this process will be negligible with respect to the emission due to the stellar feedback. First, if a UV background is present, molecular hydrogen can be destroyed by photodissociation. Even if there is no UV background, dissociation of molecular hydrogen by internal UV radiation emitted from massive stars, formed in the high density regions of the objects, is very efficient and the evolution of the objects and star formation are strongly affected (Omukai \& Nishi 1999). This regulation effect is efficient as long as the line emission of molecular hydrogen is the main cooling process, i.e. if $Z \lesssim 10^{-2} Z_{\odot}$ and $T \lesssim 8000 \mathrm{~K}$ (Nishi \& Tashiro 2000). Second, the mass of these objects is very small and therefore the amount of gas in the required thermodynamic state for the emission is very limited. Interestingly, the relative little importance of PopIII objects in terms of their radiation power in ionizing photons with respect to objects of mass above $\mathrm{M}_{H}$ has already been established by Ciardi et al. (2000). The latter objects emit a very large fraction of their binding energy in the hydrogen Ly $\alpha$ line which is therefore a much better tracer of their formation (Haiman, Spaans \& Quataert 2000).

\section{ACKNOWLEDGMENTS}

It is a pleasure to acknowledge useful discussions with $\mathrm{R}$. Nishi, F. Palla and Y. Shchekinov.

\section{REFERENCES}

Benson, A., Nusser, A., Sugiyama, N. \& Lacey, C. G. 2000, preprint (astro-ph/0002457)

Blain, A. W., Frayer, D. T., Bock, J. J. \& Scoville, N. Z. 2000, MNRAS, 313, 559

Bruscoli, M., Ferrara, A., Fabbri, R. \& Ciardi, B. 2000, MNRAS, in press (astro-ph/9911467)

Chrysostomou, A., Brand, P. W. J. L., Burton, M. D. \& Moorhouse, A. 1993, MNRAS, 265, 329

Ciardi, B., Ferrara, A., Governato, F. \& Jenkins, A. 2000, MNRAS, in press (astro-ph/9907189)

Combes, F. \& Pfenniger, D. 1997, A\&A, 327, 453

Ferrara, A. 1998, ApJ, 499, 17L

Frayer, D. T. \& Brown, R. L. 1997, ApJS, 113, 221

Haiman, Z., Rees, M. J. \& Loeb, A. 1997, ApJ, 476, 458
Haiman, Z., Spaans, M. \& Quataert, E. 2000, preprint (astro$\mathrm{ph} / 0003366)$

Gruzinov, A. \& Hu, W. 1998, ApJ, 508, 435

Knox, L., Scoccimarro, R. \& Dodelson, S. 1998, Phys. Rev. Lett., 81,2004

Marri, S. \& Ferrara, A. 1998, ApJ, 509, 43

Marri, S., Ferrara, A. \& Pozzetti, L. 2000, MNRAS, in press (astro-ph/0001469)

Nishi, R. \& Tashiro, M. 2000, ApJ, 537, in press

Oh, S. P. 1999, ApJ, 527, 160

Omukai, K. \& Nishi, R. 1999, ApJ, 518, 64

Press, W. H. \& Schechter, P. 1974, ApJ, 187, 425

Rodríguez-Fernández, N. J. et al. 2000, preprint (astro$\mathrm{ph} / 0002478)$

Shchekinov, Y. A. \& Éntél', M. B. 1985, Sov. Astron., 29, 491

Silk, J. \& Spaans, M. 1997, ApJL, 488, 79

Spitzer, L. Jr. 1978, Physical Processes in the Interstellar Medium (Wiley-Interscience: New York)

Timmermann, R. et al. 1996, A\&A, 315, 281L

Turner, J., Kirby-Docken, K. \& Dalgarno, A. 1977, ApJS, 35, 281 